\title{
Joint Energy and Performance Aware Relay Positioning in Flying Networks
}

This paper was downloaded from TechRxiv (https://www.techrxiv.org).

\section{LICENSE}

CC BY 4.0

SUBMISSION DATE / POSTED DATE

07-02-2022 / 10-02-2022

\section{CITATION}

Rodrigues, Hugo; Coelho, André; Ricardo, Manuel; Campos, Rui (2022): Joint Energy and Performance Aware Relay Positioning in Flying Networks. TechRxiv. Preprint.

https://doi.org/10.36227/techrxiv.19128008.v1

$\mathrm{DOI}$

10.36227/techrxiv.19128008.v1 


\title{
Joint Energy and Performance Aware Relay Positioning in Flying Networks
}

\author{
HUGO RODRIGUES, ANDRÉ COELHO, (Graduate Student Member, IEEE), MANUEL \\ RICARDO, (Member, IEEE), AND RUI CAMPOS, (Senior Member, IEEE) \\ INESC TEC and Faculdade de Engenharia, Universidade do Porto, 4200-465 Porto, Portugal. \\ Corresponding author: André Coelho (E-mail: andre.f.coelho@inesctec.pt).
}

\begin{abstract}
This work is co-financed by National Funds through the Portuguese funding agency, FCT - Fundação para a Ciência e a Tecnologia, within project UIDB/50014/2020 and by the European Regional Development Fund (FEDER), through the Regional Operational Programme of Lisbon (POR LISBOA 2020) and the Competitiveness and Internationalization Operational Programme (COMPETE 2020) of the Portugal 2020 framework [Project 5G with Nr. 024539 (POCI-01-0247-FEDER-024539)]. The second author also thanks the funding from FCT under the PhD grant SFRH/BD/137255/2018.
\end{abstract}

\begin{abstract}
Unmanned Aerial Vehicles (UAVs) have emerged as suitable platforms for transporting and positioning communications nodes on demand, including Wi-Fi Access Points and cellular Base Stations. This paved the way for the deployment of flying networks capable of temporarily providing wireless connectivity and reinforcing the coverage and capacity of existing networks anywhere, anytime. Several solutions have been proposed in the literature for the positioning of UAVs that act as Flying Access Points (FAPs). Yet, the positioning of Flying Communications Relays (FCRs) in charge of forwarding the traffic to/from the Internet has not received equal attention. A major challenge in flying networks is the UAVs endurance. Since the UAVs are typically powered by on-board batteries with limited capacity, whose energy is used for communications and propulsion, the UAVs need to land frequently for recharging or replacing their batteries, limiting the flying network availability. State of the art works are focused on optimizing both the flying network performance and the energy-efficiency from the communications point of view, but do not consider the energy spent for the UAV propulsion. Yet, the energy spent for communications is typically negligible when compared with the energy spent for the UAV propulsion. In order to address the FCR UAV positioning and energy-efficiency challenges, we have proposed the Energy-aware RElay Positioning (EREP) algorithm. EREP defines the trajectory and speed of the FCR UAV that minimize the energy spent for the UAV propulsion. However, since EREP considers a theoretical radio propagation model for computing the minimum Signal-to-Noise Radio (SNR) values that allow to meet the FAPs traffic demand, this may lead to network performance degradation in real-world networking scenarios, especially due to the FCR UAV movement. In this article, we propose the Energy and Performance Aware relay Positioning (EPAP) algorithm. Built upon the EREP algorithm, EPAP defines target performance-aware SNR values for the wireless links established between the FCR UAV and the FAPs and, based on that, computes the trajectory to be completed by the FCR UAV, so that the energy spent for the UAV propulsion is minimized. EPAP was evaluated in terms of both the flying network performance and the FCR UAV endurance, considering multiple networking scenarios. Simulation results show gains up to $25 \%$ in the FCR UAV endurance, while not compromising the Quality of Service offered by the flying network.
\end{abstract}

INDEX TERMS Aerial Networks, Energy-aware, Flying Networks, Performance-aware, Quality of Service, Relay Positioning, Unmanned Aerial Vehicles, UAV trajectory.

\section{INTRODUCTION}

Over the last years, the use of Unmanned Aerial Vehicles (UAVs) for a myriad of civil and military applications has increased [1, 2]. Their capability to operate virtually anywhere, their ability to hover above the ground, and their increasing capacity to carry cargo on board make UAVs suitable platforms for transporting and positioning communications nodes, including Wi-Fi Access Points and cellular Base Stations. This paved the way for the deployment of flying networks that provide wireless connectivity and 


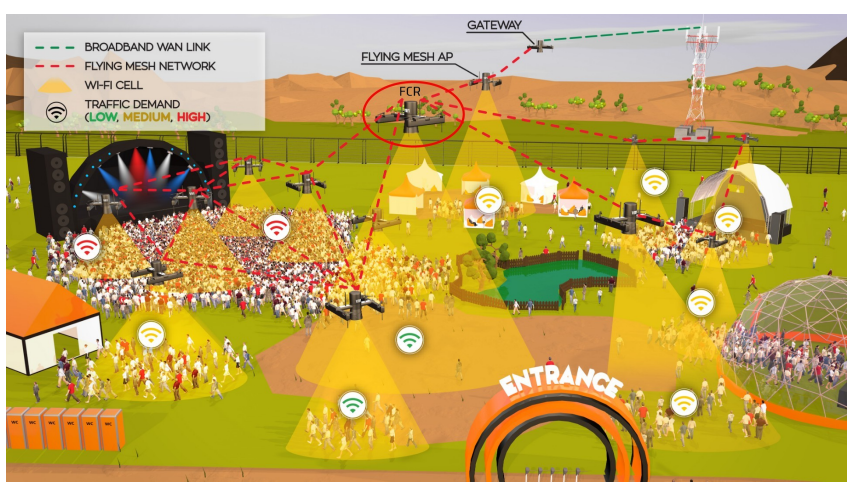

FIGURE 1: Flying Network providing Internet connectivity to the spectators in a music festival. The FCR UAV is circled in red.

reinforce the coverage and capacity of existing networks on demand, enabling the broadband Internet access in temporary events, including music festivals and disaster management scenarios [3, 4]. However, flying networks impose important challenges regarding the positioning of the UAVs, in order to meet the Quality of Service (QoS) expected by the users. In this article, we assume that two types of UAVs compose the flying network: Flying Access Points (FAPs) and a single Flying Communications Relay (FCR). In the literature, several solutions have been proposed for the positioning of FAPs, aiming at enhancing the wireless coverage, the number of ground users served [5-8], or the QoS offered [3, 9,-13]. However, the positioning of the FCR UAV has not received equal attention. The FCR UAV plays a crucial role in the flying network, as it is the communications node responsible for forwarding the traffic between the FAPs and the Internet, as depicted in Fig. 1 .

In order to address the FCR UAV positioning challenge, a traffic-aware gateway positioning (GWP) algorithm for flying networks with controlled topology was proposed in [14]. GWP considers the traffic demand of the FAPs and their positions to define in advance the positioning of the FCR UAV, taking advantage of the holistic knowledge provided by a central node. However, GWP neglects an important concern in flying networks: the energy spent for the UAV propulsion. As UAVs are not connected to the electrical grid, they typically rely on their on-board batteries, which have limited capacity. For this reason, the UAVs need to land frequently for recharging or replacing their batteries [15].

The problem occurs when the UAVs play the role of FCRs, especially in a flying network composed of only one FCR UAV. The available energy at the FCR UAV directly influences the overall performance of the flying network, since if the FCR UAV becomes unavailable due to power shortage, the FAPs will be unable to access the Internet. Therefore, the energy spent for the UAV propulsion should be taken into account when planning flying networks, in order to improve their availability. Flying networks with high availability will be able to provide connectivity for a longer time interval, thus increasing the amount of traffic that can be exchanged. This problem is identified in [16], which refers to resource management and energy-efficiency as open research challenges for using of UAVs in flying networks. A reference work on energy-efficiency in rotary-wing UAVs is presented in [17]. The authors have concluded that the power consumption when the UAVs move at low speed values is lower than when the UAVs are in the hovering state. Therefore, hovering is not the most energy-efficient UAV state.

Within this context, a joint energy and performance-aware FCR UAV positioning algorithm that meets the FAPs traffic demand while ensuring an energy-efficient UAV behavior is worthy of being considered. In order to address this challenge, in [18] we have proposed an Energy-aware RElay Positioning algorithm for flying networks, called EREP. EREP takes into account the energy spent for the UAV propulsion, as well as the positions and the traffic demand of the FAPs providing wireless connectivity to the ground users, in order to define the trajectory and speed of the FCR UAV that minimize its power consumption. Still, EREP was validated considering the wireless channel modeled by a theoretical radio propagation model only. In real-world networking scenarios, it is expected that the network performance is degraded due to SNR deviations with respect to the values obtained by means of theoretical propagation models, especially when the FCR UAV is moving to complete the trajectory defined by EREP. In order to solve this problem, we propose the Energy and Performance Aware relay Positioning (EPAP) algorithm. Built upon the EREP algorithm, EPAP defines target performance-aware SNR values for the wireless links established between the FCR UAV and the FAPs and, based on that, computes the trajectory to be completed by the FCR UAV, in order to minimize the FCR UAV energy consumption for propulsion, while ensuring the targeted flying network performance. EPAP is evaluated in terms of: i) the flying network performance; and ii) the FCR UAV endurance, considering the energy spent for the UAV propulsion in multiple networking scenarios.

The main contributions of this article are three-fold:

1) The Energy and Performance Aware relay Positioning (EPAP) algorithm, which allows to compute the trajectory to be completed by the FCR UAV, in order to minimize its energy consumption, while ensuring the targeted flying network performance.

2) Evaluation of the flying network performance achieved when the EPAP algorithm is employed by means of ns-3 simulations carried out using a realistic wireless channel model.

3) Evaluation of the FCR UAV endurance when using the EPAP algorithm, considering multiple networking scenarios composed of a different number of FAPs and average distances between them, including random networking scenarios.

The rest of this article is organized as follows. Section III presents the related work on energy and performance aware 
UAV positioning. Section $[\mathrm{III}$ describes the system model and formulates the problem addressed in this article. Section IV presents the EPAP algorithm. Section $\nabla$ presents the evaluation of the EPAP algorithm in terms of the flying network performance, and the energy consumption and endurance of the FCR UAV. SectionVI discusses the results achieved, and the pros and cons of the EPAP algorithm. Finally, SectionVII points out the main conclusions and directions for future work.

\section{RELATED WORK}

Flying networks composed of UAVs have emerged as a flexible and agile solution to provide broadband Internet access in areas where terrestrial networks are not available and when their coverage and capacity need to be enhanced. Flying networks present many advantages in a myriad of scenarios, including the possibility of an on-demand, quick deployment anywhere, anytime. Also, in flying networks the wireless links are characterized by a strong Line-of-Sight component, paving the way to use simplified radio propagation models for their planning, including the Free-Space Path Loss model [13, 19]; this represents an important advantage when compared with terrestrial networks. Moreover, UAVs have fully controlled mobility in three-dimensional (3D) space and can adaptively change their positions for reducing the Euclidean distance to the ground users, in order to improve the wireless coverage and the QoS offered. These advantages were exacerbated by the advent of small and low-cost UAVs.

\section{A. UAV ENERGY CONSUMPTION}

In flying networks made up of UAVs, energy is spent for two main tasks: communications and UAV propulsion [20]. In order to improve the energy-efficiency in communications, the literature has been focused on different communications layers: 1) network layer, by designing energy-aware routing protocols that compute the forwarding tables taking into account the energy available in the UAVs [21--23]; 2) data link layer, by improving the MAC scheme for using sleep modes when the communications nodes are in an idle state [24, 25]; and 3) physical layer, by optimizing the hardware design, and the performance of the signal transmission and processing tasks [26]. However, the energy spent for communications is typically negligible when compared with the energy spent for the UAV propulsion. For instance, an off-the-shelf IEEE 802.11n Network Interface Card has an estimated power consumption up to $2 \mathrm{~W}$ [27, 28], while the power consumption for the UAV propulsion when in hovering state is greater than 150 W, as depicted in Fig. 2 .

A reference work on joint energy-efficiency and trajectory optimization for an FCR UAV is presented in [29], where the authors propose a theoretical model to estimate the energy spent for the UAV propulsion, considering the UAV speed, direction, and acceleration. In order to maximize the UAV energy-efficiency, a circular trajectory with optimized radius and flight speed is proposed. Nevertheless, the pro-

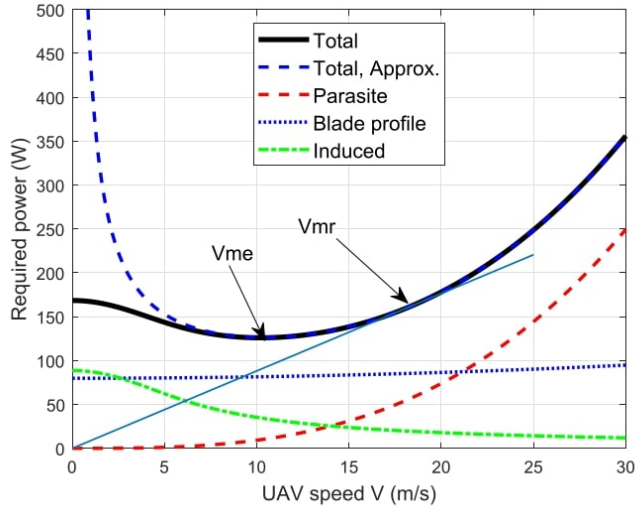

FIGURE 2: Propulsion power consumption versus UAV speed $V$ [17].

posed model targets fixed-wing UAVs and considers only one ground user.

In [30, 31], the optimal altitude for rotary-wing UAVs acting as FAPs is derived, taking into account both the energy consumption and the performance from the communications point of view. However, their authors consider UAVs in hovering state only, which is not the most efficient state when it comes to the energy spent for the UAV propulsion. This challenge was also not addressed in [32], where in addition to the UAV hovering altitude, the transmission power is jointly optimized for allocating communications resources using UAVs in a space-air-ground networking scenario.

In [33], the energy-efficient UAV deployment problem is formulated taking into account the flight dynamics and the QoS offered to the users. In terms of flight dynamics, the influence of altitude, UAV components, and payload weight in power consumption during hovering are studied. The authors concluded that the higher the UAV is, the more power it consumes, since as the altitude increases, the air density decreases. On the other hand, when the air density decreases, in order the UAV is able to maintain constant thrust, the induced air velocity needs to be increased. For this purpose, the propellers need to increase the blade tips speed, thus raising the power consumption. Finally, it is concluded that a higher payload weight leads to an increased power consumption.

In [34], an energy-aware 3D UAV deployment solution is proposed, aiming at optimizing the throughput. The tradeoff between flight altitude, energy consumption, and travel time is addressed. With respect to the power spent for the UAV propulsion, it is shown that moving the UAV at high speed values consumes more power than hovering, whereas hovering requires more power than climbing in altitude.

In [35, 36], an energy-efficiency model for multiple UAVs, taking into account the energy consumed by the UAVs and a suitable deployment approach for recharging them, is proposed, aiming at providing seamless long-term wireless connectivity. Still, the communications cells are achieved 
by positioning the UAVs in hovering state and the speed in which the UAVs move to/from the charging station is not optimized for minimizing the UAVs' energy consumption.

\section{B. UAV POSITIONING}

The literature on UAV positioning has been focused on defining the positions and trajectories of: 1) FAPs, considering the wireless coverage, the number of ground users served [5.-8], or the QoS offered to the ground users [3, 9.-13]; and 2) FCR UAVs forwarding traffic between ground nodes [37, 39]. However, existing works aim at optimizing both the flying network performance and energy-efficiency from the communications point of view [40-43], neglecting the energy spent for the UAV propulsion.

When it comes to the FAP positioning in flying networks, a reference work is proposed in [3], where the NetPlan algorithm is proposed. NetPlan is based on the Potential Fields (PF) technique, which lies on PF generators that apply forces on the FAPs; these forces are attractive to areas with high traffic demand and rejective to areas with low traffic demand. In order to improve the aggregate throughput, the FAPs closer to the users with higher traffic demand establish smaller Wi-Fi cells, whereas the remaining FAPs establish larger Wi-Fi cells so that the entire area is covered.

A UAV can also be used to extend the communications range between ground nodes. In [37], the effect in the network performance resulting from the asymmetrical positioning of an FCR UAV between two ground nodes is studied. Three possible networking scenarios are explored by moving an FCR UAV from an equidistant position between the ground nodes to a non-equidistant position. The authors concluded that: 1) the data rate of the link established with the farther ground node is reduced, while the data rate of the other link is not improved; 2) the data rate of the link established with the nearest ground node is improved without reducing the data rate of the link to the node further away; and 3 ) the date rate of the link to the nearest node is improved, while the data rate of the link established with the further apart node is decreased. Gains up to $35 \%$ in throughput were achieved, when compared with the positioning in a central position. Moreover, the altitude of the FCR UAV and the distance between the ground nodes were shown to have great influence in the throughput. However, the traffic demand of the ground nodes was not considered.

The positioning challenge for a single FCR UAV considering the traffic demand of multiple FAPs has been addressed in [14], where the GWP algorithm was proposed. Taking into consideration the future positions of the FAPs, which are defined by a FAP positioning algorithm running on a central node, and the bitrate of the generated traffic flows, the GWP algorithm aims at guaranteeing that the wireless link established between each FAP and the FCR UAV has a minimum SNR that enables the use of the minimum Modulation and Coding Scheme (MCS) index able to accommodate the traffic demand. The Free-Space Path Loss model is used to estimate the SNR of the wireless link established between each FAP and the FCR UAV, which acts as the gateway to the Internet. The maximum distance in $3 \mathrm{D}$ space corresponds to the radius of a sphere, centered at each FAP, within which the FCR UAV should be positioned. Considering all the FAPs in the flying network, the position defined for the FCR UAV corresponds to the subspace generated by the intersection of all the spheres. This position is determined by the GWP algorithm following an iterative approach, considering the transmission power of the UAVs as the fine-tuning parameter, which is successively increased until intersection between the spheres occurs. The results obtained in the performance evaluation of GWP show that it is possible to improve the overall performance of the flying network by dynamically adjusting the position of the FCR UAV, considering both the positions and traffic demand of the FAPs. However, the energy consumption of the FCR UAV is not considered by GWP, as the FCR UAV is positioned in hovering state; according to [17], hovering is not the most energy-efficient UAV state.

In order to improve the performance of GWP, we have proposed the EREP algorithm [18]. EREP allows to define the trajectory and speed of the FCR UAV that minimize its energy consumption for propulsion. However, since EREP uses a theoretical radio propagation model for determining the minimum SNR values required to meet the FAPs' traffic demand, it may lead to network performance degradation in the real-world, especially due to the FCR UAV movement. In order to address this challenge, a joint energy and performance-aware FCR UAV positioning algorithm is worthy of being considered.

\section{SYSTEM MODEL AND PROBLEM FORMULATION}

When positioning a single FCR UAV, which is the communications node responsible for forwarding traffic to/from the Internet, the traffic demand of each FAP providing wireless connectivity to the ground users should be taken into account. We assume that the information about the traffic demand is provided by a Central Station, which is a centralized node deployed anywhere in the Cloud or at the Edge of the flying network. The Central Station is in charge of defining the positions of the FAPs by running a state of the art FAPs positioning algorithm [3], thus providing a holistic knowledge about the network. In our article, the number of FAPs in the flying network is variable; however, a single FCR UAV is always considered.

When planning a flying network, the limited energy on board the UAVs should be taken into consideration, as it restricts the total amount of time the network is available. A flying network composed of UAVs with higher endurance will be able to serve the connected users for a longer time, thus increasing the network availability. In the state of the art, when it comes to UAV positioning, several solutions aim at determining the optimal positions where the FAPs and the FCR UAVs should hover. However, moving UAVs at relatively low speed values has been proven to consume less energy than hovering [44][17]. For these reasons, an algo- 


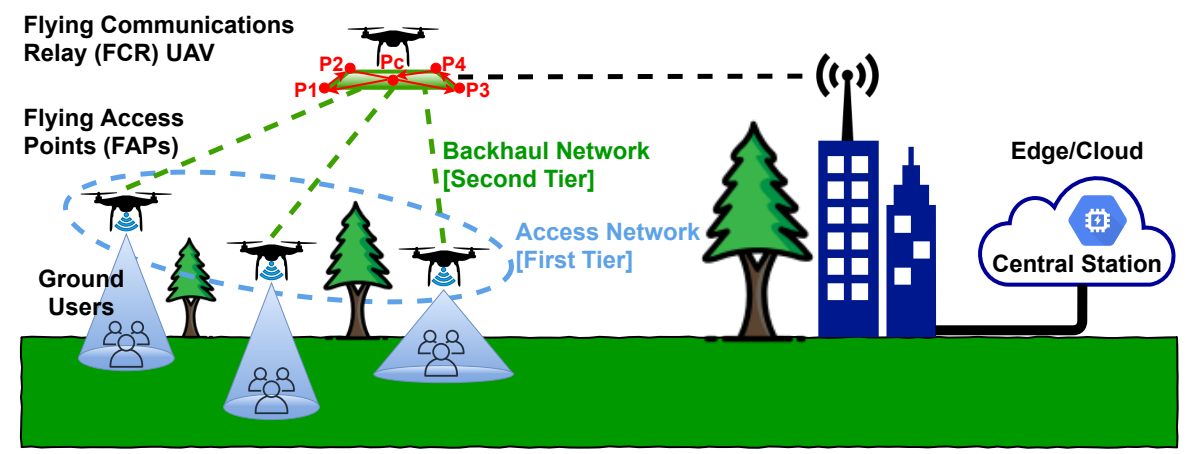

FIGURE 3: Flying network composed of two-tiers: access and backhaul networks. The FAPs, in the first tier, provide wireless connectivity to the ground users, while the FCR UAV, in the second tier, forwards the traffic to/from the Internet. The UAVs positioning over time is computed in a Central Station deployed in the Cloud or at the Edge of the flying network. An example trajectory defined by the EPAP algorithm is depicted by the red arrows.

rithm for positioning the FCR UAV taking into consideration the traffic demand of all FAPs while maintaining an energyefficient behavior is proposed herein, in order to improve the overall performance of the flying network.

We assume that the wireless links established between the FAPs and the FCR UAV are modeled by the FreeSpace Path Loss model [45], due to the strong Line-of-Sight component between UAVs flying above the ground [13, 19]. As such, the power received $P_{r}$ at the FCR UAV is computed using (1), where $P_{t}$ is the FAP transmission power, $\lambda$ is the wavelength given by the speed of light in vacuum $c$ over the carrier frequency $f$ used, and $r$ represents the distance between the FAPs and FCR UAV. We assume the maximum channel capacity is defined by the data rate associated with the MCS index used by the UAVs, which requires a minimum $S N R=P_{r} / P_{N}$. For computing $S N R$, a constant noise power $P_{N}$ is considered. The wireless medium is shared and all UAVs can listen to any other UAV. The Carrier Sense Multiple Access with Collision Avoidance (CSMA/CA) is employed for Medium Access Control (MAC).

$$
\frac{P_{r}}{P_{t}}=\left[\frac{\lambda}{4 \pi r}\right]^{2}
$$

The power $P(V)$ required by a rotor-wing UAV for propulsion while moving at speed $V$ is defined in (2) by means of three components [17]:

1) Blade profile - the power required to overcome the profile drag of the blades. It increases quadratically with $V$.

2) Induced - the power required to overcome the induced drag of the blades, which decreases with $V$.

3) Parasite - the power required to overcome the fuselage drag. It increases cubically with $V$.

$$
\begin{aligned}
P(V)= & P_{b}\left(1+\frac{3 V^{2}}{U_{\text {tip }}^{2}}\right)+P_{\text {ind }}\left(\sqrt{1+\frac{V^{4}}{4 v_{0}^{4}}}-\frac{V^{2}}{2 v_{0}^{2}}\right)^{3 / 2} \\
& +\frac{1}{2} d_{0} \rho s A V^{3}
\end{aligned}
$$

In (2), the first addend is the blade profile power in hovering state, where $U_{t i p}$ denotes the tip speed of the rotor blade. The second addend is the induced power for hovering, where $P_{\text {ind }}$ is the constant induced power, and $v_{o}$ is the mean rotor induced speed. The last addend represents the parasite component, where $d_{0}$ is the fuselage drag ratio, $\rho$ is the air density, $s$ is the rotor solidity, and $A$ is the rotor disc area. The values for the UAV related parameters can be obtained from the UAV specifications, while $\rho$ depends on the surrounding environment. In [17] has been concluded that there is an interval of UAV speed values $V$ for which the power consumed by the UAV in movement is lower than the power consumed when the UAV is in hovering state. When $V$ is set to 0 , which represents the speed value in hovering state, the power consumption $P(V)$ in 2 is equal to $P_{b}+P_{\text {ind }}$. In such case, $P(V)$ is a finite value that only depends on physical factors such as the UAV weight, air density, and rotor disc area. In turn, when the speed $V$ is positive, $P(V)$ slightly decreases for low speed values, but then it increases significantly as the speed also increases. These variations are depicted in Fig. 2, allowing to conclude that hovering is not the most energy efficient state for the FCR UAV propulsion. Among the factors that change according to the surrounding environment, the wind speed and direction have an indirect correlation with the UAV energy consumption - they only influence the speed of the UAV with respect to the air [46]. For this reason, in this article, the wind effect is neglected for both the EREP algorithm and the considered baseline.

In the formulation presented herein, a graph $G=(U, L)$ is considered for modeling the flying network. Let $U=$ $\left\{U A V_{0}, \ldots, U A V_{N-1}\right\}$ represent the set of UAVs $i$ positioned 
at $Q_{i}=\left(x_{i}, y_{i}, z_{i}\right)$ and $L \subseteq U \times U$ represent the set of directional links established between UAV $i$ and UAV $j$, with $(i, j) \in L$ and $i, j \in U$. Assuming that each $U A V_{i}$, $i \in\{1, \ldots, N-1\}$, acting as a FAP, transmits a traffic flow $F_{0, i}$ with bitrate $T_{i}$ bit/s towards $U A V_{0}$, acting as the FCR $\mathrm{UAV}$, we have a tree $T\left(U, L_{T}\right)$ that is a subgraph of $G$. This tree defines the active topology of the flying network and is composed of single-hop paths, where $L_{T} \subset L$ is the set of direct links established between each $U A V_{i}$ and $U A V_{0}$.

Considering $T$ the endurance of $U A V_{0}$, with $0 \leq t \leq T$, the problem consists in determining the trajectory $Q_{0}(t)=$ $\left(x_{0}(t), y_{0}(t), z_{0}(t)\right)$ of $U A V_{0}$, to be completed at a velocity up to the maximum value $V^{M A X}$ allowed by $U A V_{0}$, such that the power $P_{0}\left(\left\|\dot{Q_{0}}(t)\right\|\right)$ consumed by $U A V_{0}$ for propulsion is minimized. Solving this problem implies considering the maximum power $P_{0}^{M A X}$ allowed by $U A V_{0}$, while ensuring that a wireless link with high enough capacity $C_{0, i}(t)$ is available between each $U A V_{i}, i \in\{1, \ldots, N-1\}$ and $U A V_{0}$, in order to accommodate the traffic demand $T_{i}$ of $U A V_{i}$. For that purpose, the minimum $S N R_{i}$ required to induce that $U A V_{i}$ selects an MCS index with data rate higher than or equal to $T_{i}$ bit/s must be ensured. $S N R_{i}$ depends on the Euclidean distance of the wireless links established between $U A V_{i}$ and $U A V_{0}$, the carried frequency $f$, and the transmission power $P_{t}$, assumed to be equal for all the UAVs forming the flying network and set to the maximum value $P_{t}^{M A X}$ allowed for the wireless communications technology in use. Our objective function is defined in 3a.

$$
\underset{T, Q_{0}(t)}{\operatorname{minimize}} \int_{0}^{T} P_{0}\left(\left\|\dot{Q}_{0}(t)\right\|\right) d t
$$

subject to:

$$
\begin{aligned}
& 0<P_{0}\left(\left\|\dot{Q}_{0}(t)\right\|\right) \leq P_{0}^{M A X}, \forall t \in[0, T] \\
& P_{t}=P_{t}^{M A X} \\
& \left\|\dot{Q}_{0}(t)\right\| \leq V_{0}^{M A X}, \forall t \in[0, T] \\
& Q_{0}(t) \neq\left(x_{i}, y_{i}, z_{i}\right), \forall t \in[0, T], \\
& i \in\{1, \ldots, N-1\} \wedge z_{i} \geq 0, i \in\{0, \ldots, N-1\} \\
& (0, i),(i, 0) \in L_{T}, i \in\{1, \ldots, N-1\} \\
& 0<T_{i}(t) \leq C_{0, i}(t), i \in\{1, \ldots, N-1\}, \\
& \forall t \in[0, T] \\
& N-1 \\
& \sum_{i=1}^{N} C_{0, i}(t) \leq C^{M A X}, i \in\{1, \ldots, N-1\}, \\
& \forall t \in[0, T] \\
& \left(x_{0}(t)-x_{i}\right)^{2}+\left(y_{0}(t)-y_{i}\right)^{2}+\left(z_{0}(t)-z_{i}\right)^{2} \leq \\
& \left(10 \frac{P_{t}-20 \log _{10}\left(\frac{4 \pi}{\lambda}\right)-N_{0}-S N R_{i}}{20}\right)^{2}, \forall t \in[0, T], \\
& i \in\{1, \ldots, N-1\}
\end{aligned}
$$

In the defined optimization problem, the following constraints are considered for any time instant $t$ :
- $3 \mathrm{~b}$ ensures that the propulsion power $P_{0}\left(\left\|\dot{Q}_{0}(t)\right\|\right)$ enabling the operation of $U A V_{0}$ is greater than 0 and lower than or equal to the maximum power $P_{0}^{M A X}$ allowed by $U A V_{0}$.

- (3c) sets the transmission power $P_{t}$ to the maximum value $P_{t}^{M A X}$ allowed by the wireless communications technology in use.

- 3d guarantees that the speed of $U A V_{0}$ is lower than or equal to the maximum speed $V_{0}^{M A X}$ allowed by $U A V_{0}$.

- 3e ensures that the position computed for $U A V_{0}$ is different from the position of any other $U A V_{i}$ in the flying network, in order to avoid collisions. The position of each $U A V_{i}$ is defined in advance by a state of the art FAP positioning algorithm, such as the algorithm proposed in [3], which also avoids collisions for any $U A V_{i}$.

- (3f) assures that a wireless link between each $U A V_{i}$ and $U A V_{0}$ is always available in the flying network.

- 3g guarantees that the capacity in bit/s of the wireless link established between each $U A V_{i}$ and $U A V_{0}$ is greater than or equal to the traffic demand of $U A V_{i}$.

- (3h) ensures that the aggregate capacity of the wireless links established with $U A V_{0}$ is lower than or equal to the maximum capacity $C^{M A X}$ of the wireless channel used.

- (3i) ensures the target $S N R_{i}$ that enables the use of an MCS index characterized by a data rate higher than or equal to $T_{i}(t)$ bit/s. $S N R_{i}$ depends on the Euclidean distance between each $U A V_{i}$ and $U A V_{0}$, the transmission power $P_{t}$ of the UAVs, and the carrier frequency $f$ used.

Determining the solution for the problem defined in $3 \mathrm{a}$ requires computing the trajectory of $U A V_{0}$ such that the energy spent for the UAV propulsion is minimal and the capacity of the wireless links established between each $U A V_{i}$ (FAP) and $U A V_{0}$ (FCR UAV) is high enough to accommodate the traffic demand $T_{i}$ of $U A V_{i}$. The UAV trajectory planning problem has been shown in the literature to be NPhard [47, 48]. In order to achieve a solution for this problem, we propose the EPAP algorithm.

\section{ENERGY AND PERFORMANCE AWARE RELAY POSITIONING ALGORITHM}

As presented in Section III there is an interval of UAV speed values for which the power spent by the UAV for propulsion is lower than for hovering. This is the rationale of the EREP algorithm that we have proposed in [18], which was built upon the GWP algorithm [14]. GWP takes advantage of the information about the positions and traffic demand of the FAPs, provided by a state of the art FAP positioning algorithm such as the one proposed in [3], in order to enable wireless links with high enough capacity for accommodating the traffic demand of the FAPs. This position is within a 3D subspace, resulting from the intersection of the spheres centered at each FAP, with radius equal to the transmission range that ensures the minimum SNR required to induce a target 
TABLE 1: Main differences between the GWP, EREP, and EPAP algorithms. GWP positions the FCR UAV in hovering state, which leads to high energy consumption [17|, while EREP computes a trajectory to be completed by the FCR UAV at the speed that minimizes the energy spent for the UAV propulsion. EPAP improves the EREP algorithm by defining a network performance-aware trajectory for the FCR UAV.

\begin{tabular}{|c|c|c|c|}
\hline Algorithm & GWP & EREP & EPAP \\
\hline \hline $\begin{array}{c}\text { UAV state during } \\
\text { network operation }\end{array}$ & Hovering & $\begin{array}{c}\text { Moving at } \\
\text { constant speed }\end{array}$ & $\begin{array}{c}\text { Moving at } \\
\text { constant speed }\end{array}$ \\
\hline $\begin{array}{c}\text { Performance-aware } \\
\text { UAV trajectory }\end{array}$ & No & No & Yes \\
\hline $\begin{array}{c}\text { Energy consumption } \\
\text { for UAV propulsion }\end{array}$ & High & Minimum & Minimum \\
\hline
\end{tabular}

MCS index, characterized a data rate higher than or equal to the traffic demand of each FAP. Conceptually, since any position within the 3D subspace resulting from the intersection of the spheres allows to use the MCS index targeted by each FAP, the EREP algorithm improves the GWP algorithm from the energy consumption point of view by defining a trajectory within the 3D subspace, to be completed by the FCR UAV at the speed that minimizes its power consumption, instead of hovering in a fixed position. Since the movement performed by the FCR UAV to complete the defined trajectory may lead to network performance degradation in practice, due to SNR degradation, the use of an SNR margin with respect to the values computed by means of the Free-Space Path Loss model is proposed by the EPAP algorithm proposed herein. EPAP considers that when the length of the trajectory defined for the FCR UAV is long enough to induce SNR degradation, due to a wide intersection volume between the spheres centered at the FAPs, then the minimum theoretical SNR value required for the wireless link established between the FCR UAV and each FAP should be increased. This will result in a smaller intersection volume, leading to a shorter trajectory for the FCR UAV and avoiding network performance degradation due to the FCR UAV movement. The main differences between the GWP, EREP, and EPAP algorithms are presented in Table 1

The EPAP algorithm starts by determining the minimum $S N R_{i}$ that allows to induce an MCS index $\left(M C S_{i}\right)$ able to accommodate the traffic demand $T_{i}$, in bit/s, of $F A P_{i}$ (line 1 of Alg. 1). The relation between $S N R_{i}$ and the fair share of the wireless channel capacity is considered, as proposed in [14], taking into account the number of FAPs that use the same wireless channel and, thus, limiting the capacity of the wireless link achievable in practice. The fair share is defined as the data rate associated with the MCS index used by the wireless link established between each FAP and the FCR UAV over the number of FAPs sharing the medium. An illustrative example is presented in Table 2 for two FAPs, considering the IEEE 802.11ac technology with one spatial stream, 800 ns Guard Interval (GI), and $160 \mathrm{MHz}$ channel bandwidth. The minimum $S N R_{i}$ required for inducing $M C S_{i}$ imposes a minimum received power $P_{r_{i}}$ at $F A P_{i}$.
TABLE 2: Minimum SNR values required to induce the minimum and maximum IEEE 802.11ac MCS indexes, and corresponding fair share for two FAPs that use the same wireless channel [49]. The same rationale is valid for any MCS index and different number of FAPs.

\begin{tabular}{|c|c|c|}
\hline SNR (dB) & MCS data rate (Mbit/s) & Fair share (Mbit/s) \\
\hline \hline 11 & 58.5 & $\frac{58.5}{2}=29$ \\
\hline 38 & 780 & $\frac{780}{2}=390$ \\
\hline
\end{tabular}

Then, considering a constant transmission power $P_{t}$ set to $20 \mathrm{dBm}$ for all FAPs, EPAP computes the maximum transmission range $r_{i}$, so that the minimum targeted $S N R_{i}$ is ensured, as presented in Fig. 4 (line 2 of Alg. 1). In 3D space, $r_{i}$ represents the radius of a sphere centered at $F A P_{i}$. After that, EPAP finds the volume that results from the intersection of the spheres centered at each $F A P_{i}$, as illustrated in Fig. 4 . The intersection between the spheres defines the volume within which the FCR UAV can move without compromising the QoS offered. If no intersection is found, the EPAP algorithm is terminated (line 5 of Alg. 1). In order to solve the problem, a suitable approach lies on forming clusters of nearby FAPs and assign an FCR UAV to each cluster, using the EPAP algorithm to compute the trajectory for each FCR UAV independently. Yet, this challenge is left for future work.

Considering that an intersection volume is found for a single FCR UAV, the altitude corresponding to the largest area (largest 2D plane) within such volume is considered (line 9 of Alg. 1). The EPAP algorithm avoids significant changes in altitude when defining the trajectory for the FCR $\mathrm{UAV}$, in order to minimize the UAV power consumption [33. 50, 51]. The centroid of that area is the same position defined by the GWP algorithm and constitutes the waypoint where all possible trajectories for the FCR UAV pass through (line 10 of Alg. 1). EPAP computes three possible trajectories (line 11 of Alg. 1), each consisting of four waypoints (P1, P2, $\mathrm{P} 3$, and $\mathrm{P} 4)$ in addition to the centroid ( $\mathrm{Pc})$. An illustrative example for a reference networking scenario is depicted in Fig. 5. For the first trajectory (Fig. 5a), the four waypoints are located in the intersection area with the highest and lowest values of $x$, in both extremes of the $y$-axis. For the second trajectory (Fig. 5b), the corresponding four waypoints assume the highest and lowest values of $y$, in both extremes of the $x$-axis. Finally, for the third trajectory (Fig. 5c), the four waypoints have the same $x$ or $y$ coordinate as the centroid, in the extremes of the intersection area. The trajectory with the highest total sum of distances between successive waypoints, including the centroid, is selected (line 12 of Alg. 1). This allows to maximize the time the FCR UAV is moving at the speed that enables the lowest power consumption. Taking into account the minimum SNR obtained by means of the Free-Space Path Loss model, if the trajectory length is a) higher than or equal to $160 \mathrm{~m}$, then the targeted SNR is 


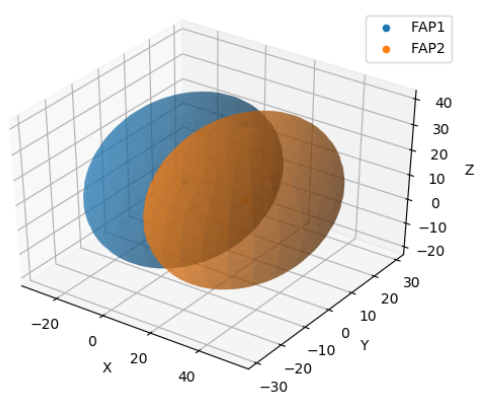

FIGURE 4: Spheres representing the transmission range of each FAP, calculated using the Free-Space Path Loss model, constrained by the SNR value that enables the selection of an MCS index compliant with the traffic demand. The intersection volume, within which the FCR UAV can move, results from the intersection of the spheres.

increased by $4 \mathrm{~dB}$ (line 13 and 14 of Alg.1); b) higher than or equal to $120 \mathrm{~m}$ but shorter than $160 \mathrm{~m}$, then the targeted SNR is increased by $3 \mathrm{~dB}$ (line 16 and 17 of Alg. 1); c) higher than or equal to $80 \mathrm{~m}$ but shorter than $120 \mathrm{~m}$, then the targeted SNR is increased by $2 \mathrm{~dB}$ (line 19 and 20 of Alg.1). In practice, this approach ensures an SNR margin with respect to the values obtained by means of the Free-Space Path Loss model or any other theoretical propagation model employed, in order to avoid network performance degradation when the FCR UAV is moving. The values considered for the SNR margin are configuration parameters in the EPAP algorithm and were obtained following a trial and error approach; their fine-tuning is left for future work.

Considering the selected trajectory (the longest trajectory in any scenario), the UAV starts at Pc and moves to P1. Afterward, it goes to P2 and then to P3, passing through $\mathrm{Pc}$. Before returning to Pc, the UAV passes through $\mathrm{P} 4$. The UAV hovers for $1 \mathrm{~s}$ at each waypoint before inverting the movement direction and proceeding to the next waypoint. The $1 \mathrm{~s}$ hovering is used as an approximation to take into account the energy consumed for changing the movement direction. This was considered in EPAP because, to the best of our knowledge, there is no model in the state of the art available to characterize the energy consumed by rotor-wing UAVs to perform such action. In the optimization problem (3), this approximation is represented by a set of consecutive equal waypoints $Q_{0}(t)$ during 1 second.

In EPAP, the transmission range that ensures the targeted SNR for the wireless link established between each FAP and the FCR UAV is represented by a sphere centered at each FAP. The volume resulting from the intersection of all spheres is depicted in Fig. 4); it is denoted by the IntersectionPoints variable in Algorithm 11. DesiredAltitude is the $z$ value in which the $x y$-plane with the largest area is located, within the intersection volume (IntersectionPoints variable). This plane enables the longest trajectory for the FCR UAV, allowing to minimize the energy consumed by the UAV for

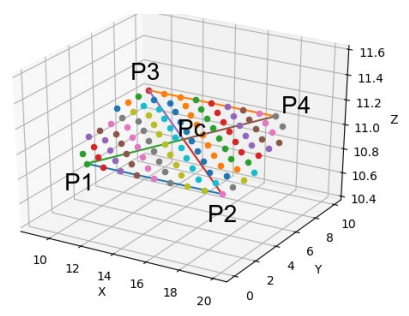

(a) Trajectory 1 .

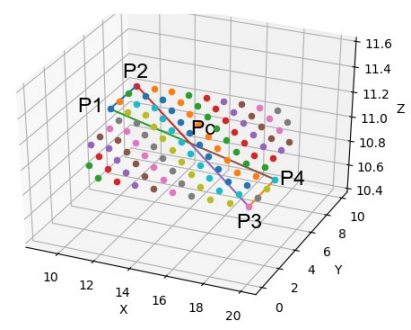

(b) Trajectory 2.

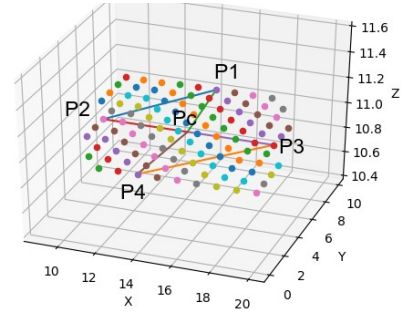

(c) Trajectory 3 .

FIGURE 5: Example trajectories defined by the EPAP algorithm.

propulsion. The centroid is the position of that plane that minimizes the Euclidean distance between the FCR UAV and the FAPs. Finally, the maximumDistance variable is the length of the longest trajectory amongst the three trajectories (cf. Fig. 5a, Fig. 5b and Fig. 5c computed by EPAP. The length of each trajectory is the sum of the Euclidean distances between the successive waypoints forming the trajectory and is calculated considering their Cartesian coordinates.

\section{EPAP EVALUATION}

In order to evaluate the FCR UAV endurance and the network performance when employing the EPAP algorithm, a set of specific networking scenarios based on the reference scenario depicted in Fig. 3 were defined; they aim at showing how the distance between the FAPs greatly influences the FCR UAV endurance. These scenarios were composed of a single FCR UAV and a variable number of FAPs positioned at extreme distances from each other: 1) FAPs close to each other; and 2) FAPs away from each other. The Cartesian coordinates of the FAPs for the considered scenarios are presented in Table 3. Moreover, in order to assess how different numbers of FAPs and the average distance between them influence the gains in the FCR UAV endurance obtained when using EPAP, networking scenarios consisting in FAPs randomly positioned were considered. In order to define the traffic demand of each FAP, we took into account an estimation for the maximum channel capacity of the wireless channel, assumed to be equal to $65 \%$ of the data rate associated with the highest MCS index possible for the network configuration employed (65\% $\times 780 \mathrm{Mbit} / \mathrm{s} \approx 500 \mathrm{Mbit} / \mathrm{s}$ ) over the number of FAPs using the same wireless channel. The $65 \%$ factor allows to consider a margin with respect to the theoretical channel capacity and take into account the inefficiency of the IEEE 802.11's MAC 


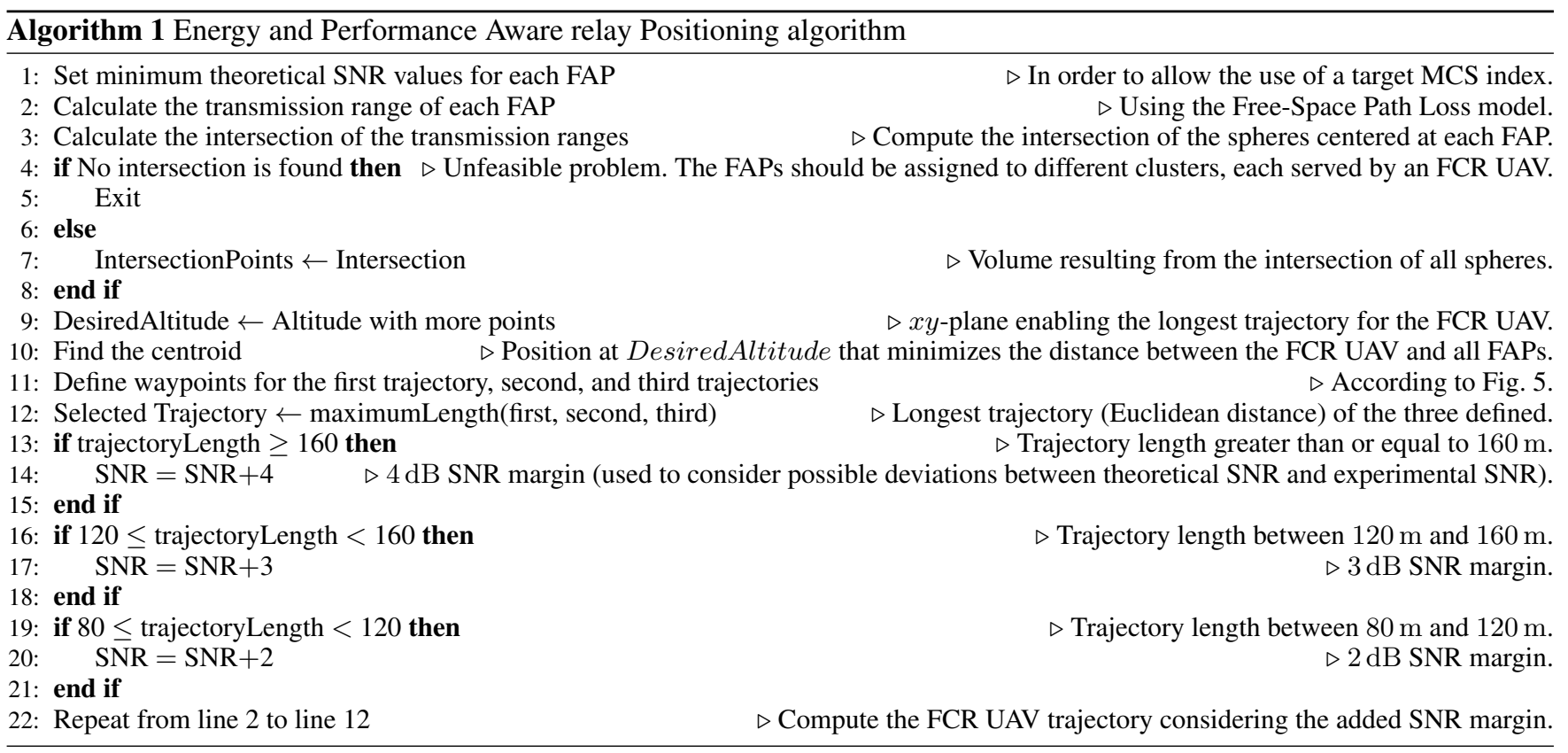

scheme observed in practice.

TABLE 3: Cartesian coordinates of the FAPs considered in the performance evaluation under extreme networking scenarios.

\begin{tabular}{|c|c|}
\hline Networking scenario & FAPs' Cartesian coordinates \\
\hline \hline 2 FAPs close to each other & $(0,0,10),(1,0,10)$ \\
\hline 2 FAPs away from each other & $(0,0,10),(29,0,10)$ \\
\hline 5 FAPs close to each other & $\begin{array}{c}(19,40,12),(1,0,10),(7,17, \\
17),(9,16,7),(10,36,13)\end{array}$ \\
\hline 5 FAPs away from each other & $\begin{array}{c}(40,38,12),(4,23,3),(13,29, \\
6),(27,3,4),(18,42,18)\end{array}$ \\
\hline & $\begin{array}{c}(20,25,18),(9,20,17),(20,13, \\
5),(24,35,13),(20,40,7),(35, \\
\text { 10 FAPs close to each other }\end{array}$ \\
$\begin{array}{c}42,12),(41,30,15),(40,25,1), \\
(14,43,17)\end{array}$ \\
\hline 10 FAPs away from each \\
other & $\begin{array}{c}(41,31,13),(39,3,0),(17,30,20,1),(12,49,7),(38, \\
23,10),(33,24,18),(25,38,9), \\
(36,20,19),(39,24,17)\end{array}$ \\
\hline
\end{tabular}

The network performance evaluation took into account two QoS metrics: 1) aggregate throughput, which consists of the average number of bits received per second by the FCR $\mathrm{UAV}$; and 2) delay, which represents the time taken by each data packet to reach the sink application of the FCR UAV, since the instant it was generated by the source application of each FAP, including queuing, transmission, and propagation delays; it was measured based on packets collected every $10 \mathrm{~ms}$, during the simulation time.

The evaluation of the EPAP algorithm under random networking scenarios was also performed, in order to assess the gains obtained in the FCR UAV endurance when using
EPAP, considering different number of FAPs and average Euclidean distances between them.

\section{A. SIMULATION SETUP}

The evaluation of both the energy consumption and endurance of the FCR UAV was evaluated by means of the UAVPowerSim simulator, originally proposed in [18] and publicly available in [52]. For the physical attributes characterizing the UAV model and the surrounding environment, we considered the values proposed in [17], including: UAV weight $W=20 \mathrm{~N}$, rotor radius $R=0.4 \mathrm{~m}$, blade angular velocity $\Omega=300 \mathrm{rad} / \mathrm{s}$, incremental correction factor to induced power $k=0.1$, profile drag coefficient $\delta=0.012$, air density $\rho=1.225 \mathrm{~kg} / \mathrm{m}^{3}$, rotor disc area $A=\pi \cdot R^{2}$ $=0.503 \mathrm{~m}^{2}$, rotor blade's tip speed $U_{t i p} \triangleq \Omega R=120 \mathrm{~m} / \mathrm{s}$, fuselage drag ratio $d_{0}=0.6$, mean rotor induced velocity in hovering state $v_{0}=\sqrt{\frac{W}{2 \rho A}}=4.03$, rotor solidity $s=0.05$, blade profile power in hovering state $P_{b} \triangleq \frac{\delta}{8} \rho s A \Omega^{3} R^{3} \approx$ 79.86, and induced power in hovering state $P_{\text {ind }} \triangleq(1+$ k) $\frac{W^{3 / 2}}{\sqrt{2 \rho A}} \approx 88.63$. When considering these values, the speed that minimizes the energy spent by the UAV for propulsion is $V \approx 10.2 \mathrm{~m} / \mathrm{s}$.

For evaluating the network performance achieved when employing the EPAP algorithm, the ns-3 simulator [53] was used. A Network Interface Card was configured on each simulated UAV in Ad Hoc mode, using the IEEE 802.11ac standard in channel 50. The traffic generated was UDP Poisson for a constant packet size of 1400 bytes. The data rate was automatically defined by the MinstrelHtWifiManager mechanism. The traffic generation was carried out during $130 \mathrm{~s}$ simulation time. The Controlled Delay (CoDel) algorithm [54], which is a queuing discipline that considers the time that packets are held in the transmission queue to discard packets, 
TABLE 4: Main simulation parameters.

\begin{tabular}{|c|c|}
\hline Parameter & Value \\
\hline UAV weight $(W)$ & $20 \mathrm{~N}$ \\
\hline Rotor radius $(R)$ & $0.4 \mathrm{~m}$ \\
\hline Blade angular velocity $(\Omega)$ & $300 \mathrm{rad} / \mathrm{s}$ \\
\hline Incremental correction factor to inducer power $(k)$ & 0.1 \\
\hline Profile drag coefficient $(\delta)$ & 0.012 \\
\hline Air density $(\rho)$ & $1.225 \mathrm{~kg} / \mathrm{m}^{3}$ \\
\hline Rotor disc area $(A)$ & $0.503 \mathrm{~m}^{2}$ \\
\hline Tip speed of the rotor blade $\left(U_{t i p}\right)$ & $120 \mathrm{~m} / \mathrm{s}$ \\
\hline Fuselage drag ratio $\left(d_{0}\right)$ & 0.6 \\
\hline Mean rotor induced velocity in hovering state $\left(v_{0}\right)$ & 4.03 \\
\hline Rotor solidity $(s)$ & 0.05 \\
\hline Blade profile power in hovering state $\left(P_{b}\right)$ & 79.86 \\
\hline Induced power in hovering state $\left(P_{i n d}\right)$ & 88.63 \\
\hline IEEE 802.11 standard & IEEE 802.11ac \\
\hline Guard Interval (GI) & $800 \mathrm{~ns}$ \\
\hline Channel bandwidth & $160 \mathrm{MHz}$ \\
\hline Spatial streams & 1 \\
\hline Maximum physical data rate & $780 \mathrm{Mbit} / \mathrm{s}$ \\
\hline Carrier frequency $(f)$ & $5180 \mathrm{MHz}$ \\
\hline Speed of light in vacuum $(c)$ & $3 \times 10^{8} \mathrm{~m} / \mathrm{s}$ \\
\hline Transmission power $\left(P_{t}\right)$ & $20 \mathrm{dBm}$ \\
\hline Noise power $\left(N_{0}\right)$ & $-85 \mathrm{dBm}$ \\
\hline Rician K-factor & $13 \mathrm{~dB}$ \\
\hline
\end{tabular}

was used; it allows to mitigate the bufferbloat problem [55]. The default parameters of CoDel in ns-3 [56] were used. The wireless channel was modeled by means of the Free-Space Path Loss model with Rician fast-fading, considering a Kfactor equal to $13 \mathrm{~dB}$, according to a realistic channel model built upon experimental results collected in an experimental testbed [13]. The Rician K-factor represents the ratio of the received power in the dominant component over the scattered power.

The main simulation parameters employed are summarized in Table 4.

\section{B. EVALUATION UNDER EXTREME NETWORKING SCENARIOS}

In the following, we present the results obtained when the EPAP algorithm was used under networking scenarios composed of FAPs at extreme distances between them. We start by evaluating EPAP under networking scenarios composed of two FAPs and comparing its performance with the EREP and GWP algorithms; then, we proceed to scenarios with five FAPs and ten FAPs.

The results for the network performance are represented by means of the Cumulative Distribution Function (CDF) for the packet delay and by the Complementary CDF (CCDF) for the aggregate throughput. The $\mathrm{CDF} F(x)$ allows to infer the percentage of collected packets with delay lower than or equal to $x$, while the CCDF $F^{\prime}(x)$ gives the percentage of simulation time during which the average throughput was greater than $x$. The results consider all values collected during 20 simulation runs for each scenario.

\section{1) Two FAPs}

In order to demonstrate the performance gains achieved by the EPAP algorithm with respect to the EREP algorithm [18]

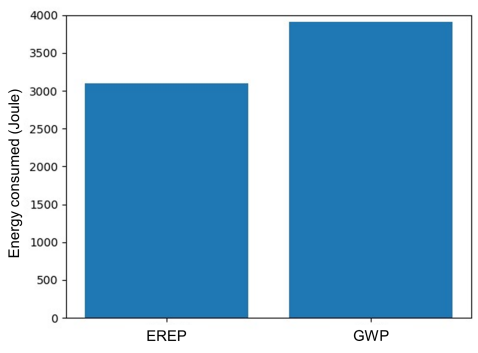

(a) Energy consumed by the FCR UAV.

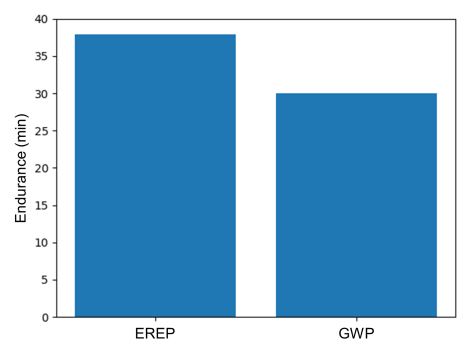

(b) FCR UAV endurance.

FIGURE 6: Energy consumption and endurance of the FCR UAV for two FAPs positioned close to each other.

and motivate the use of EPAP, we first considered a reference networking scenario consisting of two FAPs close to each other. The FAPs were generating the same amount of traffic, which was set to $250 \mathrm{Mbit} / \mathrm{s}$, enabling an intersection volume centered on the FAPs' geometric center. For this networking scenario, the third trajectory defined by EREP was chosen, since its length is much longer than the other two - trajectory 1 is $122 \mathrm{~m}$ long, trajectory 2 is also $122 \mathrm{~m}$ long, while trajectory 3 is $196 \mathrm{~m}$ long, thus allowing to achieve a higher gain in the FCR UAV endurance. Each lap to this trajectory takes a total of $23 \mathrm{~s}$ to be completed, which enables 98 laps considering the FCR UAV endurance, totaling $38 \mathrm{~min}$. To complete a lap, a total of $3096 \mathrm{~J}$ is consumed, while in hovering a total of $3913 \mathrm{~J}$ is consumed, as depicted in Fig.6a This represents a gain in the total FCR UAV endurance of 26\%, which is depicted in Fig. 6b. On the other hand, the network performance results for this scenario are depicted in Fig. 7 The $50^{\text {th }}$ percentile results show a decrease of $11 \%$ in the aggregate throughput, while an increase of $500 \%$ in the delay. The increase in delay is mainly due the high distance between the FCR UAV and the FAPs in this scenario, as the intersection volume between the two FAPs is large. When the distance between the FCR UAV and the FAPs increases, the SNR of the wireless link decreases, the capacity of the wireless channel is reduced, and the packets are held in the transmission queues longer.

The second scenario represents two FAPs positioned away from each other. Similarly, they were generating the same amount of traffic: $250 \mathrm{Mbit} / \mathrm{s}$. In this sense, the performance evaluation carried out allows to assess the influence of the distance between the FAPs for the same traffic demand. The third trajectory was selected, as it is the longest trajectory 


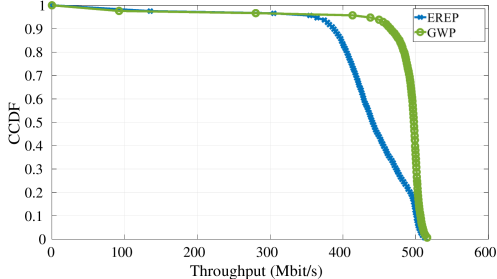

(a) Throughput CCDF.

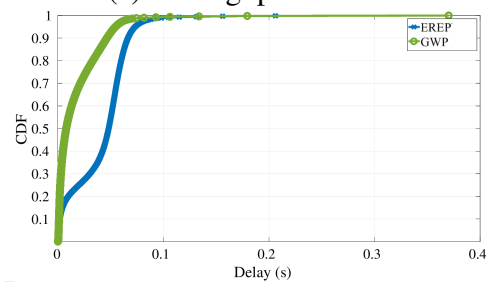

(b) Delay CDF.

FIGURE 7: Network performance results considering two FAPs positioned close to each other.

- trajectory 1 is $84 \mathrm{~m}$ long, trajectory 2 is $40.4 \mathrm{~m}$ long, and trajectory 3 is $105.7 \mathrm{~m}$ long. Each lap to the third trajectory takes a total of $14 \mathrm{~s}$ to be completed, which enables 153 laps considering the FCR UAV endurance, amounting to $37 \mathrm{~min}$. To complete a lap, a total of $1978 \mathrm{~J}$ is consumed, while in hovering state a total of $2418 \mathrm{~J}$ is consumed, as depicted in Fig. 8a This represents a gain in the FCR UAV endurance of $22 \%$, as presented in Fig. $8 \mathrm{~b}$. The network performance results are shown in Fig. 9. The $50^{\text {th }}$ percentile results show a decrease of $10 \%$ in the aggregate throughput, while an increase of $941 \%$ in the delay. The increase in the delay is justified by the same reasons as in the networking scenarios composed of two FAP close to each other, which was previously discussed.

Although the reduction in the FCR UAV energy consumption that is achieved when using the EREP algorithm, it is observable a relevant degradation in the network performance. In order to address this challenge, we propose the EPAP algorithm. As a step forward with respect to the EREP algorithm, EPAP introduces an SNR margin that ensures the targeted flying network performance, as described in Section IV] The performance evaluation of the EPAP, EREP, and GWP algorithms is depicted in Fig. 10, where it is possible to observe that the network performance results of the EPAP algorithm (in blue) are close to those obtained when the FCR UAV is in hovering state in the position defined by the GWP algorithm (in orange), while the FCR UAV energy consumption when using EPAP is substantially reduced. In order to improve the flying network performance with respect to the EREP algorithm (in green), EPAP promotes the reduction of the area within which the FCR UAV can move by imposing higher target SNR values.

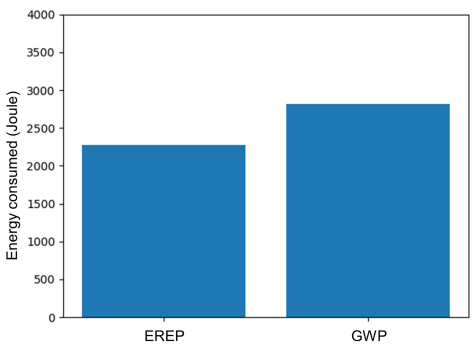

(a) Energy consumed by the FCR UAV.

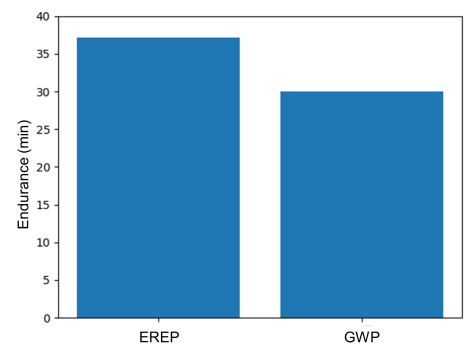

(b) FCR UAV endurance.

FIGURE 8: Energy consumption and endurance of the FCR UAV for two FAPs positioned away from each other.

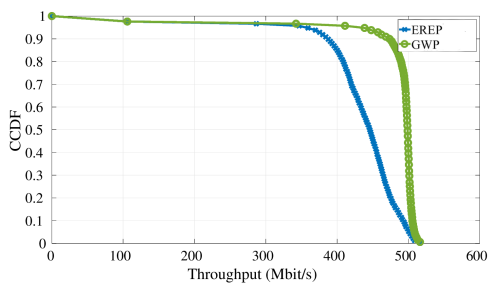

(a) Throughput CCDF.

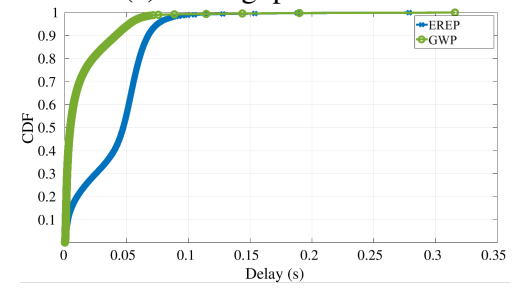

(b) Delay CDF.

FIGURE 9: Network performance results considering two FAPs positioned away from each other.

\section{2) Five FAPs}

From now on, we will focus on evaluating the EPAP algorithm against the GWP algorithm, our baseline. First, we considered a scenario composed of five FAPs close to each other, each generating $100 \mathrm{Mbit} / \mathrm{s}$. The second trajectory defined by the EPAP algorithm was chosen, since it is longer than the other two - trajectory 1 is $55 \mathrm{~m}$ long, trajectory 2 is $74 \mathrm{~m}$ long, and trajectory 3 is $66 \mathrm{~m}$ long, thus allowing to achieve a higher gain in the FCR UAV endurance. Each lap to this trajectory takes a total of $11 \mathrm{~s}$ to be completed, which enables 191 laps considering the FCR UAV endurance, corresponding to $36 \mathrm{~min}$. A total of $1584 \mathrm{~J}$ is consumed to complete 


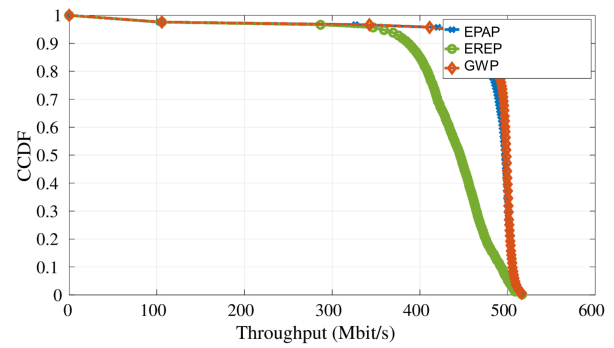

(a) Throughput CCDF.

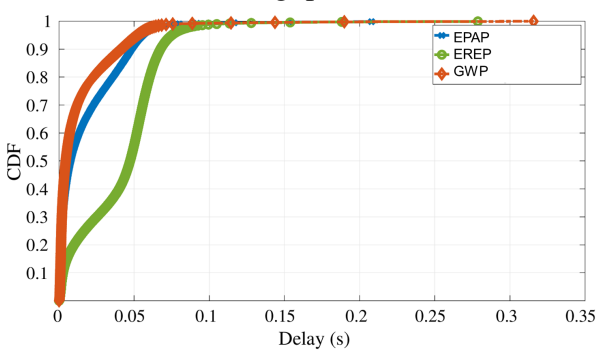

(b) Delay CDF

FIGURE 10: Performance results considering the EPAP (in blue), EREP (in green), and GWP (in orange) algorithms, for two FAPs positioned away from each other.

a lap, while in hovering a total of $1890 \mathrm{~J}$ is consumed, as depicted in Fig. 11a. This represents a gain in the FCR UAV endurance of $19 \%$, as presented in Fig. 11b The network performance results for this scenario are shown in Fig. 12 It is observable a negligible degradation for all network performance metrics when compared with the GWP algorithm - the $50^{\text {th }}$ percentile results show a decrease of $6 \%$ in the aggregate throughput, while an increase of $4 \%$ in the delay.

Then, we considered a scenario with five FAPs away from each other. The traffic demand of each FAP was set to $100 \mathrm{Mbit} / \mathrm{s}$. The third trajectory was selected, as it presents greater length than the rest - trajectory 1 is $46 \mathrm{~m}$ long, trajectory 2 is $50 \mathrm{~m}$ long, and trajectory 3 is $55 \mathrm{~m}$ long. Each lap to this trajectory takes a total of $9 \mathrm{~s}$ to be completed, which enables 224 laps for the FCR UAV endurance, amounting to $35 \mathrm{~min}$. A total of $1349 \mathrm{~J}$ is consumed to complete a lap, while in hovering a total of $1577 \mathrm{~J}$ is consumed, as depicted in Fig. 13a. This represents a gain in the FCR UAV endurance of $16 \%$, as presented in Fig. $13 \mathrm{~b}$. The network performance results are depicted in Fig. 14 Degradation is negligible for all network performance metrics: the $50^{\text {th }}$ percentile results show a decrease of $4 \%$ in the aggregate throughput, while an increase of $6 \%$ in the delay.

\section{3) Ten FAPs}

After that, we considered a scenario composed of ten FAPs close to each other, each offering $50 \mathrm{Mbit} / \mathrm{s}$. The third trajectory defined by EPAP was chosen, since its length is longer than the other two - trajectory 1 is $61 \mathrm{~m}$ long, trajectory 2 is $56 \mathrm{~m}$ long, and trajectory 3 is $79 \mathrm{~m}$ long. Each lap to this trajectory takes a total of $12 \mathrm{~s}$ to be completed,

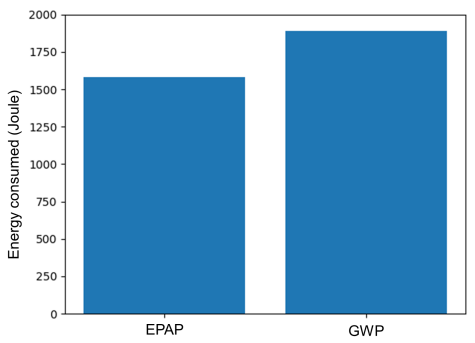

(a) Energy consumed by the FCR UAV.

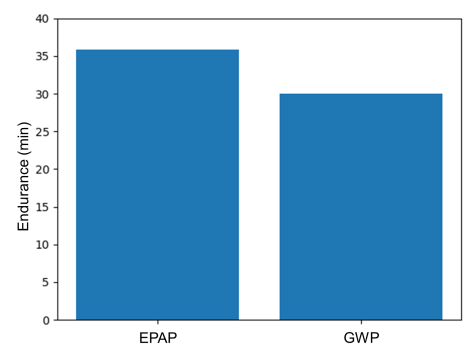

(b) FCR UAV endurance.

FIGURE 11: Energy consumption and endurance of the FCR UAV for five FAPs positioned close to each other.

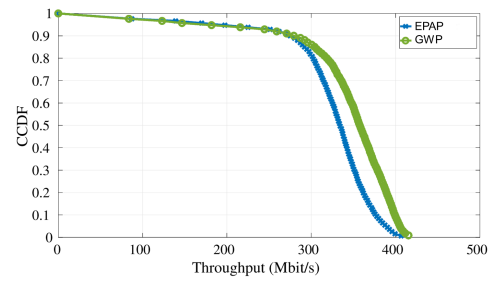

(a) Throughput CCDF.

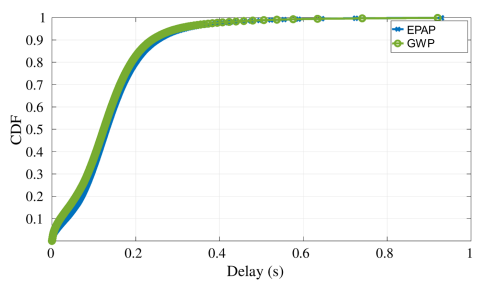

(b) Delay CDF.

FIGURE 12: Network performance results considering five FAPs positioned close to each other.

which enables 185 laps taking into account the FCR UAV endurance, amounting to $36 \mathrm{~min}$. To complete a lap, a total of $1643 \mathrm{~J}$ is consumed, while in hovering state a total of $1969 \mathrm{~J}$ is consumed, as depicted in Fig. 15a. This represents a gain in the FCR UAV endurance of $20 \%$, which is depicted in Fig. 15b. The network performance achieved when using EPAP and GWP is approximately the same, as depicted in Fig. 16 , where the $50^{\text {th }}$ percentile results show a decrease of $2 \%$ in the aggregate throughput, while an increase of $2 \%$ in the delay.

Finally, a scenario composed of ten FAPs away from each other, generating $50 \mathrm{Mbit} / \mathrm{s}$, was considered. The first trajec- 


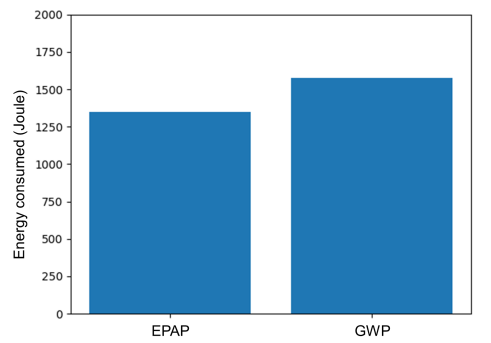

(a) Energy consumed by the FCR UAV.

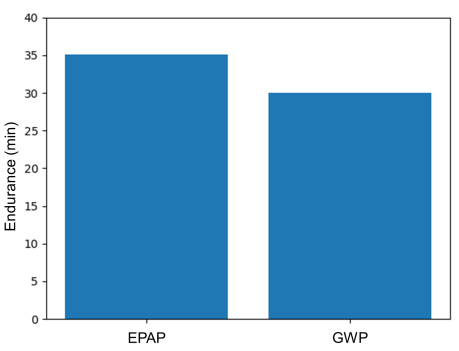

(b) FCR UAV endurance.

FIGURE 13: Energy consumption and endurance of the FCR UAV for five FAPs positioned away from each other.

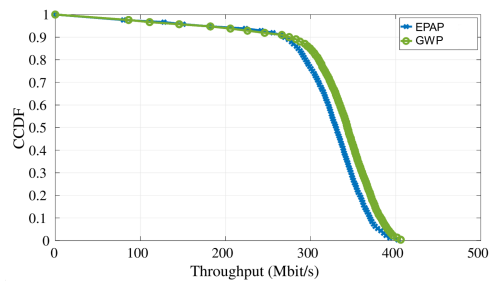

(a) Throughput CCDF.

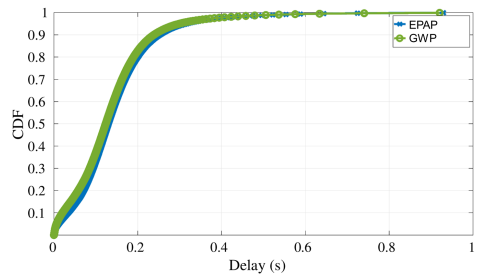

(b) Delay CDF.

FIGURE 14: Network performance results considering five FAPs positioned away from each other.

tory computed by EPAP was selected, as it presents greater length than the rest - trajectory 1 is $40 \mathrm{~m}$ long, trajectory 2 is $39 \mathrm{~m}$ long, and trajectory 3 is $19 \mathrm{~m}$ long. Each lap to this trajectory takes a total of $8 \mathrm{~s}$ to be completed, which enables 260 laps, taking into account the FCR UAV endurance equal to $34 \mathrm{~min}$. To complete a lap, a total of $1167 \mathrm{~J}$ is consumed, while in hovering a total of $1333 \mathrm{~J}$ is consumed, as depicted in Fig. 17a. This represents a gain in the FCR UAV endurance of $14 \%$, as presented in Fig. 17b The network performance results for this scenario are shown in Fig. 18. Degradation is negligible for all network performance metrics.

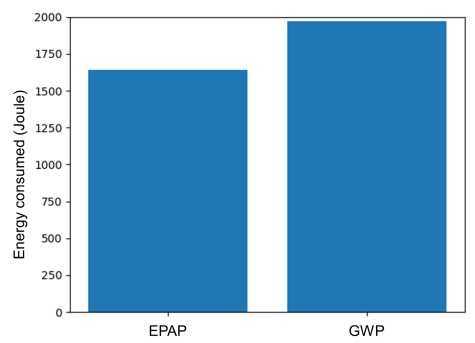

(a) Energy consumed by the FCR UAV.

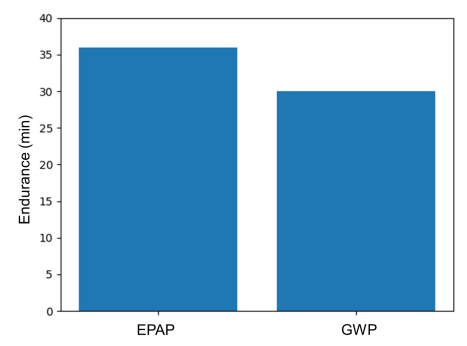

(b) FCR UAV endurance.

FIGURE 15: Energy consumption and endurance of the FCR UAV for ten FAPs positioned close to each other.

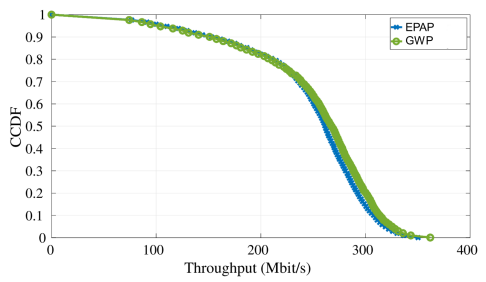

(a) Throughput CCDF.

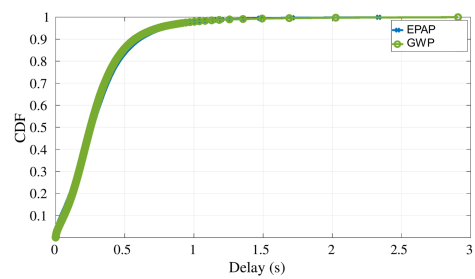

(b) Delay CDF.

FIGURE 16: Network performance results considering ten FAPs positioned close to each other.

\section{EVALUATION UNDER RANDOM NETWORKING SCENARIOS}

After evaluating the network performance achieved when using the EPAP algorithm in extreme networking scenarios, we performed the evaluation of the EPAP algorithm from the energy consumption point of view under random networking scenarios. For that purpose, we focused the evaluation on how different numbers of FAPs and the average distance between them influence the FCR UAV endurance gains obtained. The considered scenarios were generated using BonnMotion [57], a mobility scenario generation tool. For each scenario composed of the same number of FAPs, all 


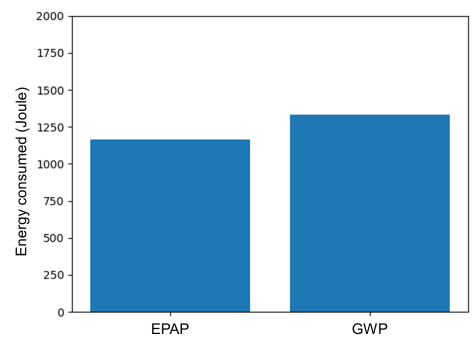

(a) Energy consumed by the FCR UAV.

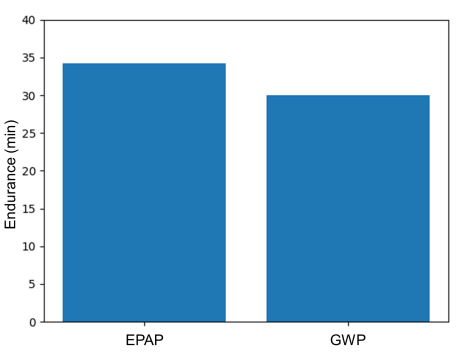

(b) FCR UAV endurance.

FIGURE 17: Energy consumption and endurance of the FCR UAV for ten FAPs positioned away from each other.

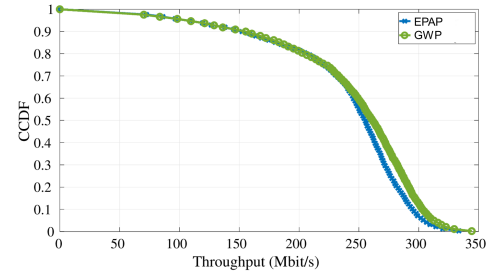

(a) Throughput CCDF.

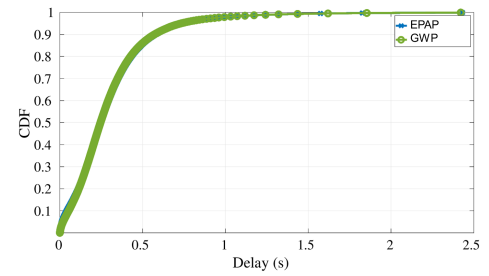

(b) Delay CDF.

FIGURE 18: Network performance results considering ten FAPs positioned away from each other.

FAPs were generating the same amount of traffic.

The results for a set of 160 random networking scenarios, considering different number of FAPs between 2 and 10, are shown in Fig. 19, where it is possible observe that they go up to approximately 25\%. A gain of $25 \%$ allows a UAV with 2-hour endurance to keep flying for 30 minutes more when compared with the baseline, which considers the UAV hovering at a fixed position. Overall, we can conclude that the higher the average distance between the FAPs, the lower the gains in the FCR UAV endurance obtained when using the EPAP algorithm, which is in line with the results obtained for the extreme networking scenarios presented in Section V-B

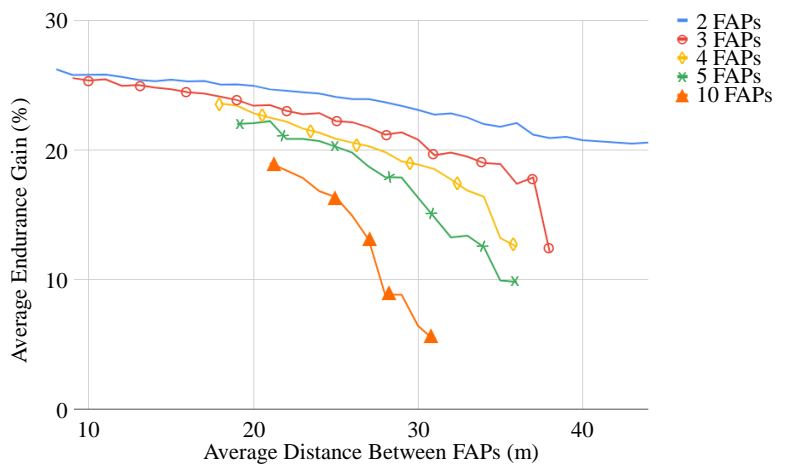

FIGURE 19: FCR UAV endurance gains for different number of FAPs and average Euclidean distances between them, considering random networking scenarios.

Moreover, for equal average distances between the FAPs, the gains in the FCR UAV endurance decrease as the number of FAPs increases. This is mainly due to the decrease in the size of the FAPs intersection volume as more FAPs are added, which leads to short trajectories for the FCR UAV. Still, EPAP always increases the FCR UAV endurance without compromising network performance.

\section{DISCUSSION}

Taking into account the rationale of the EREP algorithm proposed in [18], for networking scenarios where the FAPs are close to each other, making that the distance between each FAP and the FCR UAV increases more significantly, the network performance observed in practice is expected to be lower than when the FCR UAV is in hovering. In fact, as the distance between each FAP and the FCR UAV increases, the SNR of the wireless link decreases, the capacity of the wireless channel is reduced, and the packets are held in the transmission queues longer. This is the main drawback of the EREP algorithm. In order to address this challenge, the EPAP algorithm introduces an SNR margin, which allows to limit the intersection volume between the spheres centered at each FAP. The results presented herein clearly show an improvement in the network performance achieved when using the EPAP algorithm when compared with the EREP algorithm. In fact, the network performance results for the EPAP algorithm are close to those obtained when the FCR UAV is in hovering state, while the FCR UAV endurance is significantly improved. The use of multiple random networking scenarios and different runs in the ns-3 simulations carried out provide statistical relevance to the performance results presented.

In its current version, the EPAP algorithm is used to compute the trajectory for the FCR UAV only, as it represents a single point of failure in the flying network: the FCR UAV is the only communications node in charge of forwarding the traffic to/from the Internet. When it comes to the access network, a higher flexibility exists. When a FAP needs to land for power charging, the positioning and the transmission power of the remaining FAPs can be temporarily modified, 
allowing to provide always-on wireless connectivity to the ground users, even if the performance of the access network may be temporarily degraded. The use of the EPAP algorithm for the FAPs positioning involves addressing additional challenges, including: 1) defining a trajectory for each FAP that simultaneously allows to meet the traffic demand of the ground users and ensures that the backhaul wireless links established with the FCR UAV have high enough capacity to accommodate the traffic demand; and 2) taking into account the collision avoidance problem, since multiple FAPs are expected to move at the same time. The improvement of EPAP to compute the positioning of multiple UAVs is left for future work.

Although EPAP was evaluated in networking scenarios composed of FAPs, it can also be used to compute the trajectory of an FCR UAV used to forward traffic to/from fixed ground Wi-Fi Access Points or cellular Base Stations.

\section{CONCLUSIONS AND FUTURE WORK}

Flying networks have emerged as an agile and flexible solution for providing on-demand wireless connectivity anywhere, anytime. For that purpose, UAVs are considered suitable platforms, especially due to their high mobility in $3 \mathrm{D}$ and growing capacity to carry cargo on board, including Wi-Fi Access Points and cellular Base Stations. Yet, the positioning of the FCR UAV, which is the communications node responsible for forwarding traffic to/from the Internet, greatly affects the overall flying network performance, especially if the FAPs providing wireless connectivity to ground users have heterogeneous traffic demand. An additional challenge when planning flying networks is the energy spent for the UAV propulsion. Unlike terrestrial networks, in which the communications nodes are typically connected to the electrical grid, UAVs rely only on their on-board batteries, which have limited capacity and are drained non-linearly according to the UAV behavior. In the literature, it is shown that the energy spent for propulsion in rotary-wing UAVs decreases for low velocity values and then it starts increasing as the velocity increases, which allows to conclude that hovering is not the most energy-efficient behavior.

In this article, we proposed the EPAP algorithm, which is able to define the trajectory and speed of the FCR UAV that minimize the energy spent for the UAV propulsion, without compromising the network performance offered by the flying network. EPAP was evaluated in terms of both the flying network performance and the FCR UAV endurance, considering multiple networking scenarios and a realistic wireless channel model. The performance evaluation carried out allowed to conclude that it is possible to increase the endurance of the FCR UAV without compromising the QoS offered by the flying network when the EPAP algorithm is used.

As future work, there are some improvements that can be made to the EPAP algorithm, including: 1) the fine-tuning of the values used for the SNR margin; 2) the consideration of additional possible trajectories for the FCR UAV; 3) the de- sign and experimental evaluation of an energy consumption model for the circular movements performed by rotary-wing UAVs; and 4) evolving EPAP for multiple FCR UAVs.

\section{References}

[1] Samira Hayat, Evşen Yanmaz, and Raheeb Muzaffar. "Survey on Unmanned Aerial Vehicle Networks for Civil Applications: A Communications Viewpoint". In: IEEE Communications Surveys and Tutorials 18.4 (2016), pp. 2624-2661. ISSN: 1553877X.

[2] D. Tezza and M. Andujar. "The State-of-the-Art of Human-Drone Interaction: A Survey". In: IEEE Access 7 (2019), pp. 167438-167454.

[3] Eduardo Nuno Almeida, Rui Campos, and Manuel Ricardo. "Trafficaware multi-tier flying network: Network planning for throughput improvement". In: IEEE Wireless Communications and Networking Conference (WCNC). 2018, pp. 1-6.

[4] N. Zhao et al. "UAV-Assisted Emergency Networks in Disasters". In: IEEE Wireless Communications 26.1 (2019), pp. 45-51.

[5] Mohammad Mozaffari et al. "Drone small cells in the clouds: Design, deployment and performance analysis". In: 2015 IEEE Global Communications Conference (GLOBECOM). IEEE. 2015, pp. 1-6.

[6] Elham Kalantari et al. "Backhaul-aware robust 3D drone placement in 5G+ wireless networks". In: 2017 IEEE international conference on communications workshops (ICC workshops). IEEE. 2017, pp. 109-114.

[7] Qingqing Wu, Yong Zeng, and Rui Zhang. "Joint trajectory and communication design for multi-UAV enabled wireless networks". In: IEEE Transactions on Wireless Communications 17.3 (2018), pp. 2109-2121.

[8] Edgar Arribas, Vincenzo Mancuso, and Vicent Cholvi. "Coverage Optimization with a Dynamic Network of Drone Relays". In: IEEE Transactions on Mobile Computing (2019).

[9] Yong Zeng, Rui Zhang, and Teng Joon Lim. "Throughput maximization for UAV-enabled mobile relaying systems". In: IEEE Transactions on Communications 64.12 (2016), pp. 4983-4996.

[10] Mohamed Alzenad, Amr El-Keyi, and Halim Yanikomeroglu. "3-D placement of an unmanned aerial vehicle base station for maximum coverage of users with different QoS requirements". In: IEEE Wireless Communications Letters 7.1 (2017), pp. 38-41.

[11] E. N. Almeida et al. "A Machine Learning Based Quality of Service Estimator for Aerial Wireless Networks". In: 2019 International Conference on Wireless and Mobile Computing, Networking and Communications (WiMob). Oct. 2019, pp. 1-6.

[12] Liang Liu, Shuowen Zhang, and Rui Zhang. "CoMP in the sky: UAV placement and movement optimization for multi-user communications". In: IEEE Transactions on Communications 67.8 (2019), pp. $5645-5658$.

[13] Eduardo Nuno Almeida et al. "Joint Traffic-Aware UAV Placement and Predictive Routing for Aerial Networks". In: arXiv preprint arXiv:2004.07371 (2020).

[14] André Coelho et al. "Traffic-aware Gateway Placement for Highcapacity Flying Networks". In: 2021 IEEE 93rd Vehicular Technology Conference: VTC2021-Spring. 2021, pp. 1-6.

[15] Yong Zeng, Rui Zhang, and Teng Joon Lim. "Wireless communications with unmanned aerial vehicles: Opportunities and challenges". In: IEEE Communications Magazine 54.5 (2016), pp. 36-42.

[16] Mohammad Mozaffari et al. "A tutorial on UAVs for wireless networks: Applications, challenges, and open problems". In: IEEE Communications Surveys \& Tutorials 21.3 (2019), pp. 2334-2360.

[17] Yong Zeng, Jie Xu, and Rui Zhang. "Energy minimization for wireless communication with rotary-wing UAV". In: IEEE Transactions on Wireless Communications 18.4 (2019), pp. 2329-2345.

[18] Hugo Rodrigues et al. Energy-aware Relay Positioning in Flying Networks. 2020. arXiv: 2007.12284 [CS. NI]

[19] Aziz Altaf Khuwaja et al. "A survey of channel modeling for UAV communications". In: IEEE Communications Surveys \& Tutorials 20.4 (2018), pp. 2804-2821.

[20] Bander Alzahrani et al. "UAV assistance paradigm: State-of-theart in applications and challenges". In: Journal of Network and Computer Applications (2020), p. 102706. 
[21] Omar Sami Oubbati et al. "ECaD: Energy-efficient routing in flying ad hoc networks". In: International Journal of Communication Systems 32.18 (2019), e4156.

[22] Farhan Aadil et al. "Energy aware cluster-based routing in flying adhoc networks". In: Sensors 18.5 (2018), p. 1413.

[23] Abdel Ilah Alshabtat et al. "Low latency routing algorithm for unmanned aerial vehicles ad-hoc networks". In: International Journal of Electrical and Computer Engineering 6.1 (2010), pp. 48-54.

[24] Qiang Fan et al. "A multi-hop energy-efficient sleeping MAC protocol based on TDMA scheduling for wireless mesh sensor networks" In: Journal of Networks 7.9 (2012), p. 1355.

[25] Xue Yang and Nitin H Vaidya. "A wakeup scheme for sensor networks: Achieving balance between energy saving and end-toend delay". In: Proceedings. RTAS 2004. 10th IEEE Real-Time and Embedded Technology and Applications Symposium, 2004. IEEE. 2004, pp. 19-26.

[26] Mohammad Mozaffari et al. "Wireless communication using unmanned aerial vehicles (UAVs): Optimal transport theory for hover time optimization". In: IEEE Transactions on Wireless Communications 16.12 (2017), pp. 8052-8066.

[27] Daniel Halperin et al. "Demystifying 802.11n Power Consumption". In: Proceedings of the 2010 International Conference on Power Aware Computing and Systems. HotPower' 10. USENIX Association. Vancouver, BC, Canada, 2010, pp. 1-5.

[28] F. Sousa et al. "Green Wireless Video Sensor Networks Using Low Power Out-of-Band Signalling". In: IEEE Access 6 (2018), pp. 30024-30038

[29] Yong Zeng and Rui Zhang. "Energy-efficient UAV communication with trajectory optimization". In: IEEE Transactions on Wireless Communications 16.6 (2017), pp. 3747-3760.

[30] Nithin Babu, Constantinos B Papadias, and Petar Popovski. "EnergyEfficient 3D Deployment of Aerial Access Points in a UAV Communication System". In: IEEE Communications Letters (2020).

[31] N. Babu et al. "Energy Efficient Altitude Optimization of an Aerial Access Point". In: 2020 IEEE 31st Annual International Symposium on Personal, Indoor and Mobile Radio Communications. 2020, pp. 1 7.

[32] Jingjing Wang et al. "Joint UAV Hovering Altitude and Power Control for Space-Air-Ground IoT Networks". In: IEEE Internet of Things Journal 6.2 (2019), pp. 1741-1753.

[33] Uygar Demir et al. "Energy-Efficient Rotary-Wing UAV Deployment Under Flight Dynamics and QoS Constraints". In: 2019 IEEE International Black Sea Conference on Communications and Networking (BlackSeaCom). 2019, pp. 1-5.

[34] Shih-Fan Chou, Ai-Chun Pang, and Ya-Ju Yu. "Energy-Aware 3D Unmanned Aerial Vehicle Deployment for Network Throughput Optimization". In: IEEE Transactions on Wireless Communications 19.1 (2019), pp. 563-578

[35] Xiaowei Li et al. "A Near-Optimal UAV-Aided Radio Coverage Strategy for Dense Urban Areas". In: IEEE Transactions on Vehicular Technology 68.9 (2019), pp. 9098-9109.

[36] Xiaowei Li et al. "Rechargeable Multi-UAV Aided Seamless Coverage for QoS-Guaranteed IoT Networks". In: IEEE Internet of Things Journal 6.6 (2019), pp. 10902-10914.

[37] Erlend Larsen, Lars Landmark, and Øivind Kure. "Optimal UAV relay positions in multi-rate networks". In: IEEE, 2017 Wireless Days. 2017, pp. 8-14

[38] Xijian Zhong et al. "Deployment Optimization of UAV Relay for Malfunctioning Base Station: Model-Free Approaches". In: IEEE Transactions on Vehicular Technology 68.12 (Dec. 2019), pp. 1197111984. ISSN: 1939-9359.

[39] Xijian Zhong et al. "Joint Optimization of Relay Deployment, Channel Allocation, and Relay Assignment for UAVs-Aided D2D Networks". In: IEEE/ACM Transactions on Networking 28.2 (2020), pp. 804-817.

[40] Kai Li et al. "EPLA: Energy-balancing packets scheduling for airborne relaying networks". In: 2015 IEEE International Conference on Communications (ICC). IEEE. 2015, pp. 6246-6251.

[41] Kai $\mathrm{Li}$ et al. "Energy-efficient cooperative relaying for unmanned aerial vehicles". In: IEEE Transactions on Mobile Computing 15.6 (2015), pp. 1377-1386.

[42] Kai Li et al. "Energy efficient legitimate wireless surveillance of UAV communications". In: IEEE Transactions on Vehicular Technology 68.3 (2019), pp. 2283-2293.
[43] Zhen Qin et al. "Trajectory Planning for Data Collection of EnergyConstrained Heterogeneous UAVs". In: Sensors 19.22 (2019), p. 4884.

[44] Qingqing Wu, Liang Liu, and Rui Zhang. "Fundamental trade-offs in communication and trajectory design for UAV-enabled wireless network". In: IEEE Wireless Communications 26.1 (2019), pp. 3644.

[45] Andrea. Goldsmith. Wireless communications. Cambridge university press, 2005.

[46] Amila Thibbotuwawa et al. "Factors Affecting Energy Consumption of Unmanned Aerial Vehicles: An Analysis of How Energy Consumption Changes in Relation to UAV Routing". In: Information Systems Architecture and Technology: Proceedings of 39th International Conference on Information Systems Architecture and Technology - ISAT 2018. Ed. by Jerzy Światek, Leszek Borzemski, and Zofia Wilimowska. Cham: Springer International Publishing, 2019, pp. 228-238. ISBN: 978-3-319-99996-8.

[47] Cheng Zhan, Yong Zeng, and Rui Zhang. "Trajectory design for distributed estimation in UAV-enabled wireless sensor network". In: IEEE Transactions on Vehicular Technology 67.10 (2018), pp. $10155-10159$.

[48] Moataz Samir et al. "UAV trajectory planning for data collection from time-constrained IoT devices". In: IEEE Transactions on Wireless Communications 19.1 (2019), pp. 34-46.

[49] WLAN Pros. MCS Index chart - 802.11ac - VHT. https://www. wlanpros.com/mcs-index-charts/ (Accessed on 02/12/2021). 2021.

[50] Dimitrios Zorbas et al. "Optimal drone placement and cost-efficient target coverage". In: Journal of Network and Computer Applications 75 (2016), pp. 16-31.

[51] Hasini Viranga Abeywickrama et al. "Comprehensive energy consumption model for unmanned aerial vehicles, based on empirical studies of battery performance". In: IEEE Access 6 (2018), pp. 58383-58394.

[52] UAV Power Simulator. https://github.com/rhugo97/UAV- PowerSimulator (Accessed on 17/12/2021).

[53] NS-3. Network Simulator. URL: https://www.nsnam.org/

[54] K. Nichols and V. Jacobson. Controlling queue delay. Queue, vol. 10, 2012.

[55] Jim Gettys and Kathleen Nichols. "Bufferbloat: Dark Buffers in the Internet: Networks without Effective AQM May Again Be Vulnerable to Congestion Collapse." In: Queue 9.11 (Nov. 2011). https : //dx.doi.org/10.1145/2063166.2071893 pp. 40-54. ISSN: 15427730

[56] CoDel queue disc. https://www.nsnam.org/docs/models/html/codel. html (Accessed on 17/12/2021).

[57] Nils Aschenbruck et al. "BonnMotion: a mobility scenario generation and analysis tool". In: Proceedings of the 3rd international ICST conference on simulation tools and techniques. Proceedings of the 3rd international ICST conference on simulation tools and techniques. 2010, pp. 1-10. 